\title{
Fever in the returning traveller: A forgotten culprit
}

\author{
Karim Ladak MD, Nicole Sitzer MD, Ahraaz Wyne MD, Bahareh Ghadaki MD, Ameen Patel MB
}

\section{CASE PRESENTATION}

A 74-year-old woman presented to the emergency department after blood cultures drawn by her family physician four days previously grew Gram-negative bacilli. She had recently returned from a three-month trip to Afghanistan to visit friends and relatives, and admitted to "eating like a local" while there.

During the final two weeks of her stay in Afghanistan, she experienced fevers, chills, malaise, night sweats, myalgias and diffuse abdominal pain. She was admitted to hospital there and received two weeks of an unspecified intravenous antibiotic without clinical amelioration. On returning to Canada, her symptoms persisted for a third week and, despite multiple clinical assessments, no diagnosis was established.

While originally born in Afghanistan, the patient had lived in Canada for nearly two decades. She had not taken many dietary precautions during her trip, and admitted to consuming both cow's and goat's milk. Furthermore, she denied any exposure to animals or slaughterhouses.

On examination, her temperature was $38.9^{\circ} \mathrm{C}$. Her vital signs were otherwise within normal limits. She had mild abdominal tenderness, but no hepatosplenomegaly or lymphadenopathy.

Preliminary laboratory investigations demonstrated an elevated C-reactive protein (CRP) level $(13.4 \mathrm{mg} / \mathrm{L}$; normal $<5 \mathrm{mg} / \mathrm{L})$ and a mild transaminitis. A complete blood count, glucose, electrolytes and creatinine levels were normal. Chest and abdominal $\mathrm{x}$-rays were normal.

Based on her clinical presentation and Gram-negative bacilli bacteremia, she was treated for presumed typhoid.

On hospital day 3 her cultures speciated something unexpected...

\section{DIAGNOSIS}

Blood cultures speciated Brucella melitensis, and therapy was changed to gentamycin and doxycycline. Given Brucella's propensity to metastasize, a white blood cell scan and transthoracic echocardiogram were obtained, both of which were normal. While in hospital she received a total of seven days of gentamycin (260 mg intravenously daily) and 11 days of doxycycline (100 mg orally twice per day).

She defervesced on day 6 of hospitalization and her symptoms resolved completely shortly thereafter. She was discharged on five additional weeks of doxycycline, and remained well when seen in follow-up after completion of her antibiotic course.

\section{DISCUSSION}

Physicians should be familiar with the most prevalent pathogens causing fever in returning travellers. Despite the eradication of brucellosis from Canadian livestock in 1989 (1), it remains the most common bacterial zoonosis globally (2). Brucella is endemic in the Middle East, Central Asia, parts of Africa, Spain and Latin America, with $>500,000$ new cases annually (2). In an international 42,000-patient study conducted by the GeoSentinel Surveillance Program, it was one of the top 10 etiologies of fever in a returning traveller, particularly in those returning from North Africa and the Middle East (3). Additional pathogens from these regions included hepatitis A virus, Plasmodium falciparum, Salmonella typhi and dengue virus.

The most frequent mode of transmission is via consumption of unpasteurized dairy products. However, direct contact with animal tissue and inhalation of aerosolized particles can also lead to brucellosis (4). Once infected, the bacteria can disseminate hematogenously to virtually any organ system (4).

Brucella is a facultative intracellular, Gram-negative coccobacillus (5). Four terrestrial species are pathogenic in humans. B melitensis, whose preferred host is sheep, goats and camels, causes $>90 \%$ of human infections (6).

In Canada, the main occupational group at risk is laboratory staff, particularly because of the low infectious transmittable dose $\left(<10^{2}\right.$ organisms), and because brucellosis is often unsuspected when sent for culture $(2,6)$. There are several case reports of laboratoryacquired infection occurring in Canada (7). In the appropriate clinical context, maintaining a high index of suspicion can promote the necessary communication with laboratory personnel and implementation of safety precautions (7).

Clinically protean, Brucella is a 'major mimicker', and ranges from asymptomatic disease to severe multiorgan involvement (8). A retrospective study of 1028 patients reported fevers, arthralgias, fatigue, back pain and sweating to be the most common symptoms in acute disease. Malodorous perspiration is almost pathognomonic for brucellosis (4). The most common finding on physical examination is hepatosplenomegaly (20.6\%).

One-third of cases are complicated by localized infections, predominantly osteoarticular disease (eg, spondyloarthritis) and epididymo-orchitis (8). Hepatic involvement, neurobrucellosis and endocarditis $(<1 \%)$ can also occur $(2,4)$. If left untreated, brucellosis can persist into a debilitating state resembling chronic fatigue syndrome (8). Furthermore, it precipitates first-trimester abortions in up to $40 \%$ of pregnancies (9). However, despite Brucella's considerable morbidity, its mortality is a meager $0.1 \%$, and is almost exclusively secondary to endocarditis and neurobrucellosis (10).

Diagnosing brucellosis is challenging. Cultures are the only definitive test; however, prolonged incubation is required, and yields vary from $15 \%$ to $70 \%(4,6)$. Serology is, therefore, the preferred diagnostic modality. Serum agglutination testing is the cornerstone of diagnosis internationally. ELISA and indirect fluorescent antibody testing are better for complicated and chronic cases because these tests have a higher sensitivity and specificity. Molecular methods, such as polymerase chain reaction, have significant potential; however, they are not yet widely recommended $(4,6,8)$. A reasonable approach would be culture of blood, bone marrow or other relevant tissue, accompanied by two serological tests (6).

Elevated CRP level is the most common laboratory abnormality and can aid in diagnosis, prognostication and monitoring (2). For example, our patient had an elevated CRP level $(13.4 \mathrm{mg} / \mathrm{L})$ at the time of presentation, which normalized after completion of treatment.

Department of Internal Medicine, McMaster University, Hamilton, Ontario

Correspondence: Dr Karim Ladak, Department of Internal Medicine, McMaster University, 1280 Main Street West, HSC-3W10, Hamilton,

Ontario L8S 4K1. Telephone 905-962-2786, fax 905-529-0066, e-mail karim.ladak@medportal.ca 
Additionally, interleukin-2 was shown in a small prospective pediatric study to correlate inversely with disease resolution and may be recommended as a convalescent marker in the future (9).

The recommended antibiotic therapy of brucellosis has remained largely unchanged over the past several decades (9). Prolonged dual antibiotic therapy is recommended to avoid resistance and achieve adequate intracellular concentrations (9). According to a recent metaanalysis assessing optimal therapy for uncomplicated brucellosis, the most effective treatment regimen is a six-week course of doxycycline (100 mg orally twice per day) combined with an aminoglycoside such as gentamycin $(5 \mathrm{mg} / \mathrm{kg}$ intravenously daily) for seven days. This was more successful than oral doxycycline-rifampicin coadministration, which is currently the most widely used strategy internationally (11). Other strategies under investigation include doxycycline monotherapy, and doxycycline-trimethoprim-sulfamethoxazole and rifampicinquinolone cotherapies $(10,11)$. The efficacy of triple therapy in uncomplicated brucellosis remains unclear (11). Barriers to treatment include patient compliance, particularly because symptoms resolve

\section{REFERENCES}

1. Government of Canada. Canadian Food Inspection Agency. <www.inspection.gc.ca/animals/terrestrial-animals/diseases/reportable/ brucellosis/fact-sheet/eng/1305673222206/1305673334337> (Accessed November 18, 2013).

2. Buzgan T, Karahocagil MK, Irmak H, et al. Clinical manifestations and complications in 1028 cases of brucellosis: A retrospective evaluation and review of the literature. Int J Inf Dis 2010;14:e469-78.

3. Leder K, Torresi J, Libman M, et al. GeoSentinel surveillance of illness in returned travelers, 2007-2011. Ann Intern Med 2013;158:456-68

4. Pappas G, Akritidis N, Bosilkovski M, Tsianos E. Brucellosis. N Engl J Med 2005;352:2325-36.

5. Hatipoglu CA, Bilgin G, Tulek N, Kosar U. Pulmonary involvement in brucellosis. Can J Infect 2005;51:116-9. within a few days of commencing therapy, in addition to the logistical challenges of prolonged intravenous access for aminoglycosides, and a $10 \%$ relapse rate, which necessitates close follow-up $(10,11)$.

\section{CONCLUSION}

We present a case of fever in a returning traveller that remained undiagnosed until blood cultures speciated B melitensis. We suspect our patient was infected after consuming unpasteurized dairy products in Afghanistan because she had no obvious alternative etiologies of infection. She was successfully treated with dual doxycyclinegentamycin therapy for six weeks.

Physicians should maintain a high index of suspicion for the most common pathogens presenting as fever in a returning traveller. Pathogens common to the area(s) of travel should be identified and clinical probability should direct specific testing.

Additionally, communication between health care providers and laboratory personnel is essential to promote safe handling of the specimen and help to prevent workplace exposure to Brucella.

6. Araj GF. Update on laboratory diagnosis of human brucellosis. Int J Antimicrob Agents 2010;36(Suppl 1):S12-S17.

7. Robichaud S, Libman M, Behr M, Rubin E. Prevention of laboratory-acquired brucellosis. Clin Inf Dis 2004;38:e119-22.

8. Kilic A, Metan G, Alp E. Clinical presentations and diagnosis of brucellosis. Recent Pat Antiinfect Drug Discov 2013;8:34-41.

9. Memish ZA, Balkhy HH. Brucellosis and international travel. J Travel Med 2004;11:49-55.

10. Solera J. Update on brucellosis: Therapeutic challenges. Int J Antimicrob Agents 2010:36(Suppl 1):S18-S20.

11. Del Pozo JS, Solera J. Systematic review and meta-analysis of randomized clinical trials in the treatment of human brucellosis. PLoS One 2012;7:e32090. 


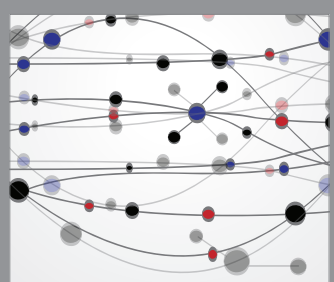

The Scientific World Journal
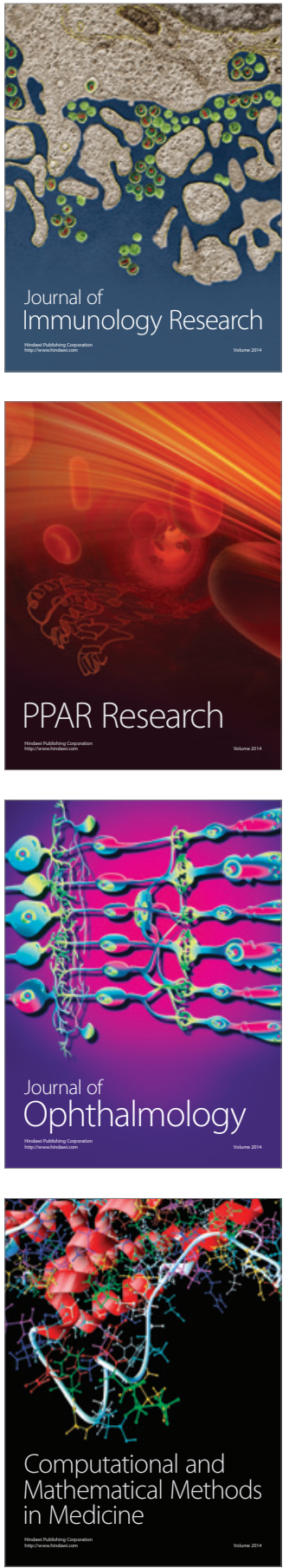

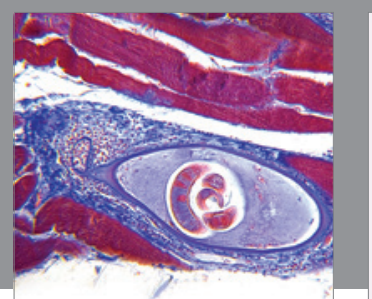

Gastroenterology Research and Practice

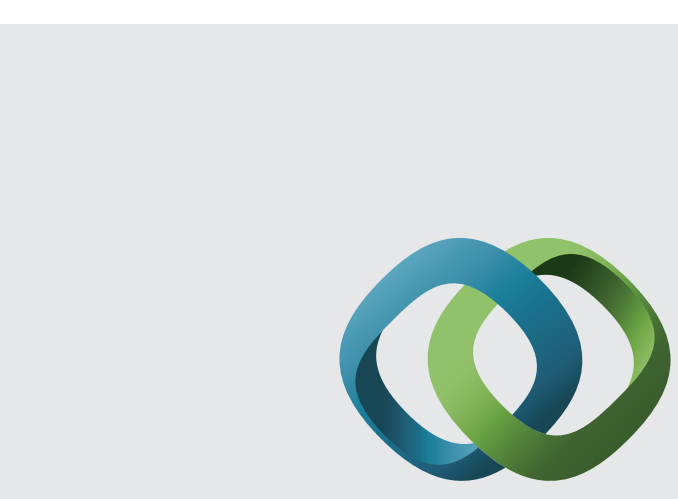

\section{Hindawi}

Submit your manuscripts at

http://www.hindawi.com
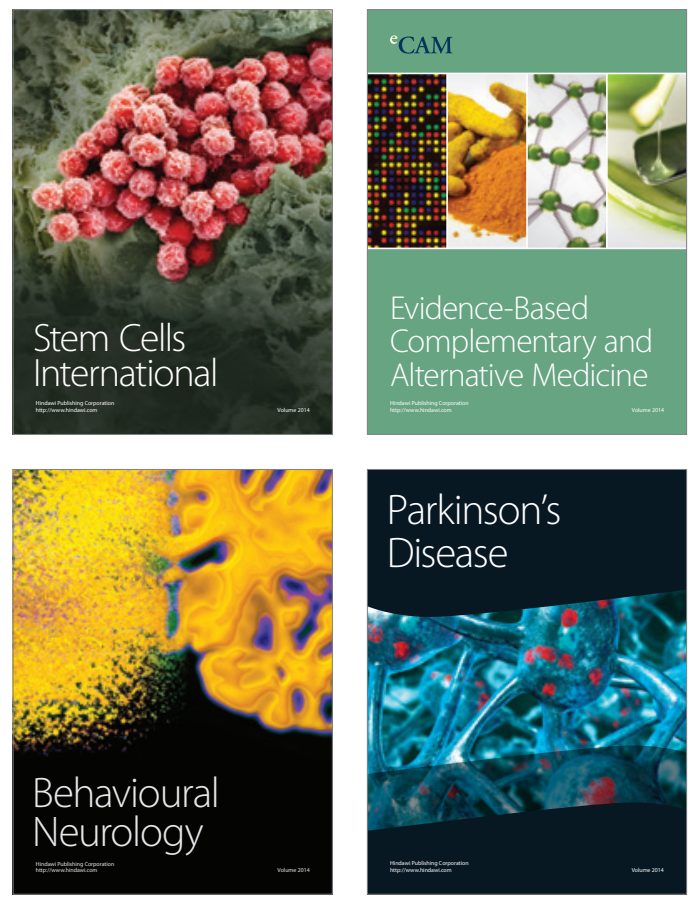
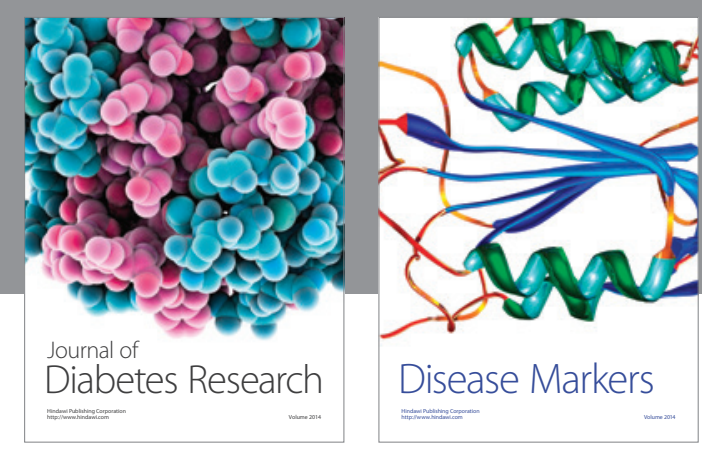

Disease Markers
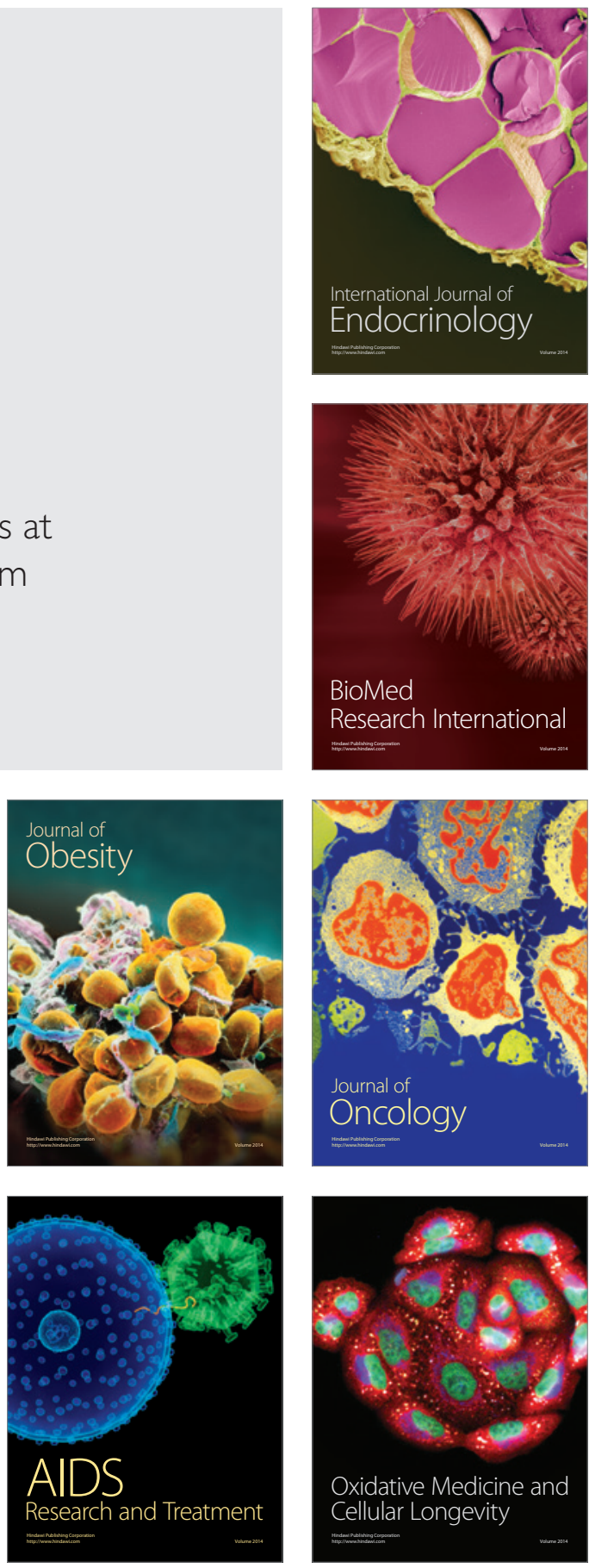